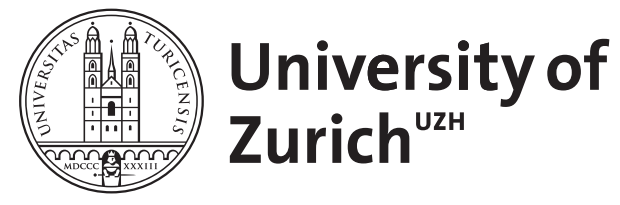

Zurich Open Repository and Archive

University of Zurich

University Library

Strickhofstrasse 39

CH-8057 Zurich

www.zora.uzh.ch

Year: 2013

The Grossman model after 40 years: response to Robert Kaestner

Zweifel, Peter

DOI: https://doi.org/10.1007/s10198-012-0450-3

Posted at the Zurich Open Repository and Archive, University of Zurich

ZORA URL: https://doi.org/10.5167/uzh-156534

Journal Article

Published Version

Originally published at:

Zweifel, Peter (2013). The Grossman model after 40 years: response to Robert Kaestner. European Journal of Health Economics, 14(2):361-362.

DOI: https://doi.org/10.1007/s10198-012-0450-3 


\title{
The Grossman model after 40 years: response to Robert Kaestner
}

\author{
Peter Zweifel
}

Published online: 3 January 2013

(c) Springer-Verlag Berlin Heidelberg 2012

The Editorial introducing the August 2012 issue of this Journal contained a critical review of the Grossman model (MGM henceforth). The Reply by Robert Kaestner in this issue starts with an impressive list of his preventive efforts ranging from jogging to having a rectal exam. All very well, but does this mean that his behavior is always guided by the kind of long-term planning that underlies the MGM?

Criticism of the MGM can be summed up in one simple, humble statement of the man in the street, "When I'm sick, I need to see the doctor".

In economic language, this is a statement about the elasticity of substitution (EOS). It claims the EOS to be (close) to zero in the sick state. This implies that the mix of inputs is dominated by medical care rather than one's own preventive effort regardless of the price of medical care relative to (mainly) one's own opportunity cost of time. The lay person's statement is silent about the EOS in the healthy state; presumably, it does not exclude a nonzero value. If so, it implies state-dependence of the EOS.

Robert Kaestner's examples designed to support the MGM would be more convincing if he were to claim that when falling ill, he would continue to combine inputs to the production of health in the same way as at present (actually, according to the MGM he should even make more effort of his own since illness presumably lowers the opportunity cost of his time while leaving the price of medical care unchanged). Also, total investment in health

This reply refers to the article available at doi:10.1007/s10198-012-451-2.

P. Zweifel $(\bowtie)$

Department of Economics, University of Zurich,

Zurich, Switzerland

e-mail: peter.zweifel@econ.uzh.ch should decrease at least in the case of a serious illness when time to death suddenly becomes short, reducing the present value of returns to investment. Neither prediction is borne out by the data.

First, there is a tradition of research that focuses on health care expenditure (HCE) in isolation (see, e.g., Manning et al. [2]). Yet according to the MGM with its state-independent EOS, a two-factor model that simultaneously explains own effort and HCE should always be specified. Moreover, using cross section data, no more than about $20 \%$ of the variance in HCE can be explained using observable personal characteristics, environmental factors, and insurance information. The coefficient of determination increases to about $50 \%$ if lagged HCE is included in the regression, but that means specifying a stochastic process-exactly the alternative sketched towards the end of the Editorial. Finally, much of the effort directed at empirically estimating HCE has been directed to its stochastic component using generalized linear models. This calls for adding a non-normal error term to the systematic, non-stochastic part of the regression equation. Usually, the Gamma distribution is found to fit best because it can accommodate the heavy skewness characterizing the distribution of HCE. Note that, conceptually, the MGM seeks to explain total HCE as measured. However, outliers in HCE of the observed magnitudes do not sit well with an investment process that involves own effort and HCE regardless of the health state the individual is in. Of course, Robert Kaestner is free to disqualify the results of the research cited above as being the outcome of 'questionable research design' (quote from his Reply).

Second, as to the influence of time to death, there is a considerable body of evidence suggesting that it is the main driver of HCE. In fact, HCE is found to just about explode towards the end of life even when the endogeneity of 
longevity with regard to HCE is controlled for [1]. Of course, Robert Kaestner is again free to dismiss this research as reflecting 'questionable research design'. Yet it may be noted that longevity amounts to nothing but the convolution (multiplication in the unlikely case of independence) of short-term survival probabilities. Therefore, the evidence cited by him linking preventive effort to longevity may very well be the outcome of sequential short-term optimization seeking to achieve a target value of the probability to be in good health (in the limit, to survive). As sketched in the Editorial, this stochastic formulation emphasizing the short run also predicts adjustment behavior. In the healthy state, the probability of being healthy turns out to be equal to one ex post, (slightly) above target-contrary to the MGM which disallows an 'overshooting' of the desired health stock. The natural way to adjust is to (slightly) cut down on preventive effort. In the sick state, this probability turns out to be zero, way below target for most. The natural adjustment is to use a lot of HCE in order to get back to the desired probability of being healthy. Sounds familiar?

Also, having learned from the MGM that HCE constitutes a long-run investment in health, just about any lay person would predict that the closer one believes to be to death, the shorter the estimated payback period and the weaker the incentive to invest by spending on HCE. As shown above, the data tell otherwise. However, when the individual considered is described as still seeing a possibility to attain his or her desired probability of being healthy tomorrow (of surviving in the limit), the same person likely would modify his or her prediction in favor of substantial spending on HCE.
On a final note, Robert Kaestner manages to disqualify the Editorial ten times on no more than two pages, using expressions ranging from 'flawed' to 'glaring weakness'. This aggressive style is reminiscent of a cleric who seeks to defend the Absolute Truth against a heretic. Scientific debate, at least in the view of this writer, revolves around consistency in theorizing and, most importantly, empirical evidence. Towards the end of his Reply, Robert Kaestner does offer a formulation designed to shore up the MGM. Unfortunately, it is of the same capital-adjustment type that was specified by Wagstaff [3] but rejected by the data as shown in the Editorial. Once more, that finding can be qualified as being the outcome of 'questionable research design'. Conversely, the common man's perception invoked here of course does not constitute systematic empirical evidence. Yet economic theorizing that is seriously at odds with the common man's view is unlikely to stand the test of time. Up to the readers of this Journal to judge ...

\section{References}

1. Felder, S., Werblow, A., Zweifel, P.: Do red herrings swim in circles? Controlling for the endogeneity of time to death. J. Health Econ. 29(2), 205-212 (2010)

2. Manning, W.G., Basu, A., Mullahy, J.: Generalized modeling approaches to risk adjustment of skewed outcomes data. J. Health Econ. 24(3), 465-488 (2005)

3. Wagstaff, A.: The demand for health: some new empirical evidence. J. Health Econ. 5(3), 195-233 (1986) 